\title{
The Web Lift and Posterior Pull for the Aging Face
}

\author{
Waleed H. Ezzat, M.D. ${ }^{1}$ Chiara Andretto Amodeo, M.D. ${ }^{2}$ Gregory S. Keller, M.D., F.A.C.S. ${ }^{3}$ \\ ${ }^{1}$ Department of Otolaryngology-Head and Neck Surgery, Boston \\ University School of Medicine, Boston, Massachusetts \\ 2 Department of Plastic and Reconstructive Surgery, University of \\ Pavia, "Salvatore Maugeri" Foundation Research and Care Institute, \\ Pavia, Italy \\ ${ }^{3}$ Department of Surgery, Division of Head and Neck Surgery, David \\ Geffen School of Medicine, University of California-Los Angeles, Los \\ Angeles, California \\ Facial Plast Surg 2012;28:126-134.

\begin{abstract}
Address for correspondence and reprint requests Gregory S. Keller, M.D., F.A.C.S., 221 W. Pueblo Street, Suite A, Santa Barbara, CA 93105 (e-mail: drgregorykeller@gmail.com).
\end{abstract}

Treatment of the aging neck is a balance between beauty and the morbidity of the surgery. A prominent trend in cervical rhytidectomy, with or without facial rhytidectomy, is a tendency to open the neck to directly remove or tighten sagging structures.

A popular method of tightening neck structures is the "corset platysmaplasty" ${ }^{1}$ and its variations. In this procedure, the platysma muscle laxity is overcome by progressively tightening the left and right platysma muscles together in the midline through an anterior incision. Wide undermining of the neck and placement of drains is part and parcel of the corset platysmaplasty.

Tightening of the neck via a corset platysmaplasty is primarily achieved with an anterior involution of the platysma muscle and precludes a great deal of backward platysma and skin pull and skin excision. As a result, procedures, such as the corset platysmaplasty, rely on the aging skin to contract and readhere to the underlying tightened subcutaneous structure. Tight adherence of skin to the tightened underlying structure may only be partially successful, producing some laxity of the skin particularly with the patient in the "head-down" position.

Attempts at treating the anterior neck with closed techniques, suspension sutures, and liposuction (with or without laser) do not, in the minds of many practitioners, adequately treat many of the problems seen in the anterior neck. Issues such as enlarged digastric muscles, subplatysmal fat collections, tightened cervicoplatysmal ligaments, short hyoidmental distance, submandibular gland ptosis, and platysma bands often require more aggressive approaches with additional potential for morbidity such as wide undermining, tissue resection, and tightening procedures.. In fact, due to the number of anatomic differences, the authors' have added ultrasound assessment of the submental area to their
Issue Theme Management of the Lower Third of the Face and Neck; Guest Editor, Phillip R. Langsdon, M.D., F.A.C.S.
Copyright @ $\odot 2012$ by Thieme Medical Publishers, Inc., 333 Seventh Avenue, New York, NY 10001, USA. Tel: +1(212) 584-4662.
DOI http://dx.doi.org/ 10.1055/s-0032-1305794. ISSN 0736-6825. 
preoperative assessment armamentarium, as previously published. $^{2}$

Corset platysmaplasty, as defined by Feldman, ${ }^{1}$ has become a "gold standard" for treatment of the neck. Corset platysmaplasty is used by many surgeons (including the senior author) when preoperative assessment of the aging neck indicates to the surgeon that aging has produced the various pathologies listed above.

Various methods of preoperative assessment of the aging neck are part of basic surgical knowledge. Palpation of the neck is one method. If the neck is "soft" and does not become hard when the patient grimaces and tightens their platysma, the neck pathology is probably limited to supraplatysmal fat with skin and platysma laxity. Many practitioners, in this instance, feel that liposuction and a posterior neck-lift may be all that is necessary. ${ }^{3}$ Some might add some form of anterior dissection and platysma suturing to this treatment recipe to avoid the "uncovering" of platysma banding by the liposuction, although others have relied on direct excision of tissue. ${ }^{4}$ Palpation that reveals a "tight" neck, resistant to upward pressure by the examiner (the senior author refers to this as "tension neck"), is indicative that the neck needs to be opened and sagging structures addressed.

Recently, a percutaneous suspension procedure (iGuide technique, first described by G. Mueller, marketed by Black and Black Surgical, Tucker, GA) has been proposed for closed treatment of the neck. ${ }^{5}$ After liposuction tunneling, the technique involves the percutaneous placement of an interdigitating suture across the submental and submandibular triangles of the neck. Tightening of the skin and platysma is achieved by hooking the ligamentous attachments between the dermis and the platysma. The sutures produce a "web" of suture that, when tightened (like the tightening of a shoelace), reestablishes the "hammock" suspension of the fallen neck (-Fig. 1).

Although the authors found this treatment alone to be valuable in the younger patient with structural neck sagging, older patients, particularly those with "platysma banding," required a posterior platysmaplasty and neck-lift to achieve a high-quality result. This article evaluates the initial results of the percutaneous suspension when used with the authors' method of combining it with a posterior neck-lift.

\section{Methods}

Our institution's investigational review board approved the retrospective protocol. We conducted a retrospective review of cases performed by the senior author (G.S.K.) from March 2010 through July 2010. Male subjects were excluded. Subjects were included if they either underwent concomitant rhytidectomy, a neck-lift combined with other cosmetic procedures, or solely a neck-lift. These subjects were entered in a database that included age, length of follow-up, satisfaction scores, and cephalometric data.
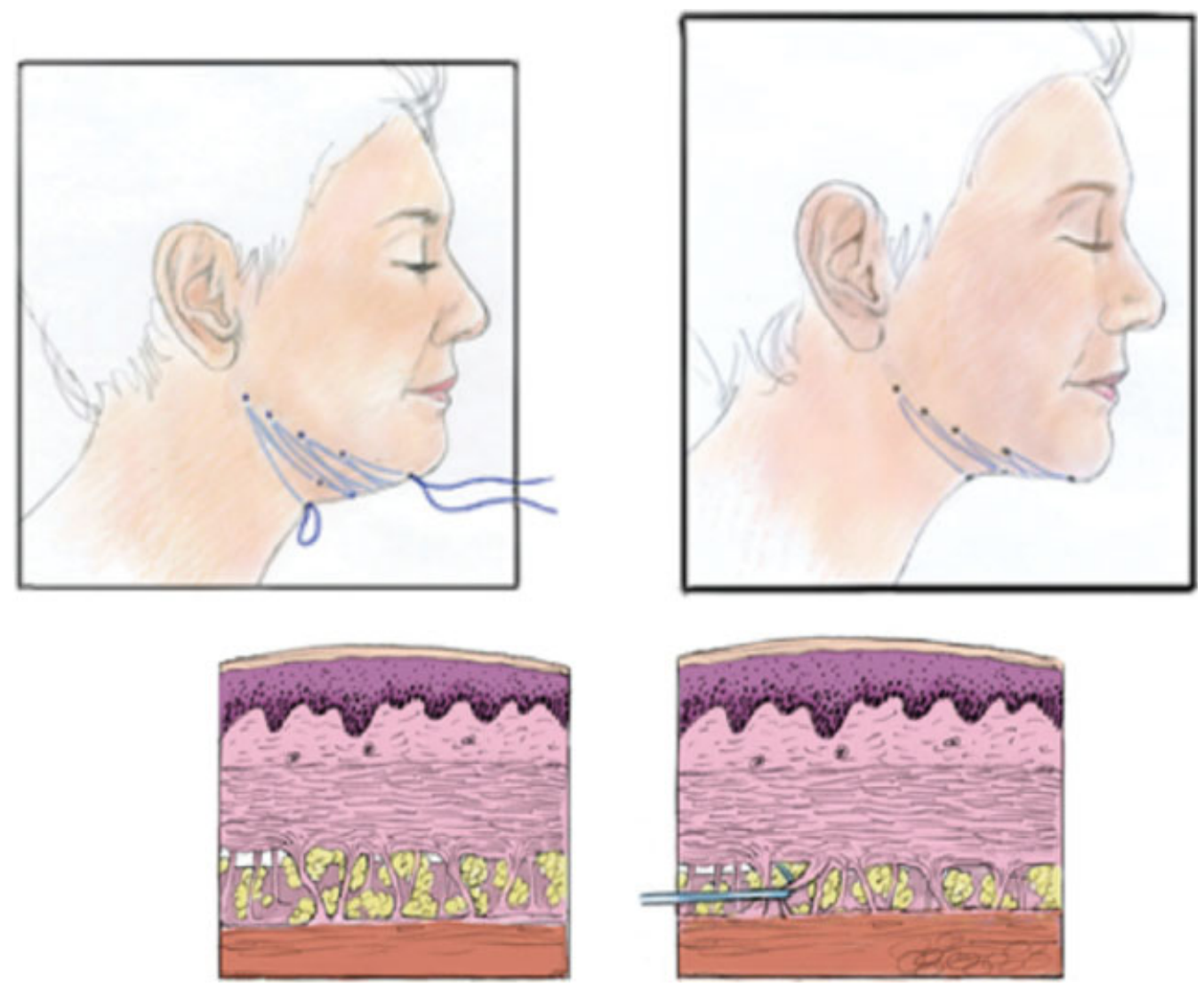

Figure 1 Placement of percutaneous sutures across the submentum (top). This suture hooks the ligamentous attachments between the dermis and platysma (bottom). 


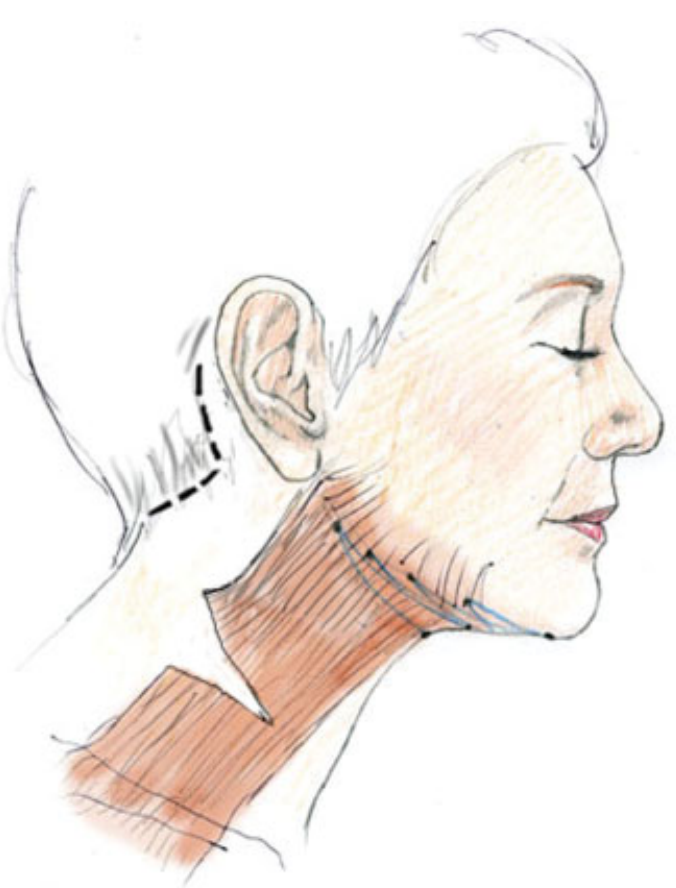

Figure 2 A full-thickness cut across the platysma muscle at the level of the cricoid is necessary for addressing platysmal banding.

\section{Procedure}

The posterior platysma lift incision follows our standard lines for aging face procedures. Cervicomental liposuction is performed through a small incision just posterior to the submental crease. The skin incisions for this technique vary, depending on the type of neck-lift that is to be performed.

For the younger individual, the only incisions that may be required are those utilized for liposuction. Older patients with significant sagging of the neck, or those with platysma bands (which the senior author refers to as the " 11 " sign) that extend beyond the hyoid, require a posterior platysmaplasty.

The incisions for posterior platysmaplasty also vary, depending on the amount of excess skin, platysma bands, and neck sagging. For patients without the need for significant skin excision, the postauricular incision is a small elliptical resection in the lower postauricular area. For patients with a greater amount of excess skin, the postauricular incisions may extend into the posterior hairline.

The extent of the posterior platysmaplasty varies with the patients. For those in need of only a lesser degree of neck tightening, a plication of the platysma to the mastoid fascia or the ligament of Lore might suffice. Greater amounts of tightening are performed according to the surgeon's preference and experience.

For patients with significant bands that extend below the hyoid, a full posterior platysmaplasty is required, as the thick platysma bands or those that extend lower than the hyothyroid complex will not respond to the hammock technique alone. In these cases, undermining is performed over the posterior neck and the sternocleidomastoid muscle fascia and extended about $2 \mathrm{~cm}$ over the posterior platysma muscle. At the level of the cricothyroid space, skin undermining is extended in a tunnel to the midline.

The platysma muscle is then separated from the sternocleidomastoid muscle, entering the fascial pocket between the superficial and deep fascia of the neck. Undermining superiorly under the platysma is only $\sim 2 \mathrm{~cm}$ until the level of the cricothyroid space, being careful to avoid the external jugular system and the cervical sensory nerve branches. A horizontal incision is performed across the platysma at the level of the cricoid.

The platysma free edges are then sutured to the mastoid fascia superiorly. Below this, the platysma edges are sutured to the sternocleidomastoid fascia to avoid extensive upward retraction of the muscle. Any skin excision is then performed in standard fashion.

The preformed template is now centered on the menton, and the markings are made along the inferior border of the mandible on each side. Small stab incisions are placed at these markings and a suture is percutaneously passed in the subcutaneous plane, thus forming of a web spanning the submandibular and submental triangles. The ends of the suture are brought through the submental incision and are tied down to accentuate the mandibular border, lift and support the submentum, and deepen the cervicomental angle (-Fig. 2). The liposuction incision is closed, along with the skin flaps posteriorly, and our standard face-lift dressing is applied.

\section{Patient Satisfaction Scores}

As part of their postoperative course, patients were presented with a brief questionnaire as to their satisfaction with the outcome of the procedure. They were also asked to rate the outcome on a 4-point scale (4 representing "very satisfied" outcome and 1 representing "not satisfied"). Further opportunity was allowed for additional comments as to their postoperative course. Scores and significant comments were entered in our databases.

\section{Cephalometric Analysis}

Preoperative photographs were compared with 1-month postoperative photographs using Mirror Suite software (Canfield Imaging Systems, Fairfield, NJ) on a Windows operating system. Lateral views taken in the Frankfort horizontal plane were utilized. A modified form of the hyomental distance was calculated in centimeters as the distance from the menton to the skin overlying the hyoid with the head in the Frankfort horizontal plane. The cervicomental angle was also measured as the angle formed by two linear lines extending from the menton toward the hyoid and another extending along the anterior neck. The angle these two lines formed when they met along the anterior neck skin was calculated in degrees (-Fig. 3).

\section{Statistical Analysis}

Statistical analysis was performed using Microsoft Excel software (Microsoft, Inc., Seattle, WA). Pre and postoperative hyomental distances and cervicomental angles were compared using a $t$ test. A p value of less than or equal to 0.05 was considered significant. 


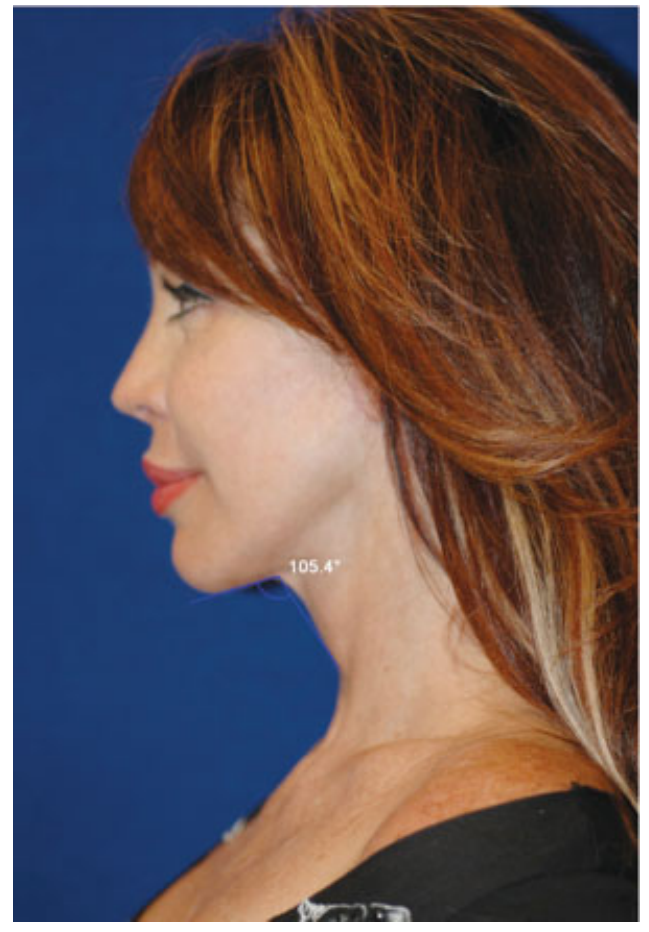

Figure 3 Cephalometric analysis of the cervicomental angle. (c) Copyright 2011 Dr. Gregory S. Keller, M.D.

\section{Results}

Thirty-eight female subjects were identified having this procedure done, either alone or in conjunction with other facial rejuvenation procedures. Of these, 13 subjects did not have follow-up photography available for analysis, leaving 25 subjects who underwent cephalometric analysis. Seventeen patients underwent neck-lift with rhytidectomy; the other eight underwent the neck-lift procedure alone. The average age of procedure was 57.9 years (range 24 to 70). Thirteen subjects were available to answer our subjective questionnaire. The average time of follow-up for subjective outcomes was 33 weeks (range 25 to 44 ).

Preoperatively, the average hyomental distance was measured at $8.5 \mathrm{~cm}$ (range 2.8 to $12.9 \mathrm{~cm}$ ) and the average cervicomental angle was calculated at 134.8 degrees (range 103.4 to 159.7 degrees). Postoperatively, both parameters had statistically significant changes. The hyomental distance increased to an average of $10.4 \mathrm{~cm}$ (range 4.7 to $15.3 \mathrm{~cm}$; $p=0.009$ ) and the cervicomental angle decreased to an average of 122.8 degrees (range 96.1 to 146.7 degrees; $p=0.002$; - Table 1 ). The overall satisfaction rate was $92.3 \%$ with an average score of 3.4 (range 1 to 4 ). One subject was unhappy with their result and stated they wish to have revision surgery. Another was satisfied with their initial result, but required revision surgery.

\section{Discussion}

The ideal characteristics that define a youthful neck should include a well-defined inferior border of the mandible, a smooth and contoured submentum, and skin that elegantly drapes the landmarks of the neck. These parameters are achieved with procedures that, in part, deepen the cervicomental angle and increase the hyomental distance. Overall, we were able to attain a significant reduction of the cervicomental angle and increased hyomental distance (-Figs. 4, 5, 6).

Of the 25 subjects who underwent analysis in our study, we achieved a satisfaction rate of $92.3 \%$. Only one patient reported being unsatisfied with her result. Another was satisfied initially but then experienced snapping of the suture spanning across her neck after undergoing rigorous activity only 3 weeks after her procedure.

The majority of subjects underwent concomitant rhytidectomy, and procedure time for this technique was not measured separately. However, we can subjectively report that our operative time spent on the neck was reduced. The combination procedure produced minimal morbidity of the neck that was primarily related to the liposuction procedure itself and suction drainage was not necessary (-Fig. 7).

There are many techniques currently utilized to address the aging neck. At one end of the spectrum lie the noninvasive procedures, utilizing ultrasound or radiofrequency energy to achieve tightening of the skin. We have seen minimal improvements from these devices with multiple administrations. Thus, we reserve this technique for patients who have well-defined borders and lines but are requesting minimal skin tightening or touch-ups.

For patients who require more invasive procedures to address the aging neck, a lateral platysmal pull, with or without a corset platysmaplasty for platysmal banding, is typically utilized by many surgeons. ${ }^{6}$ There are many inherent problems that can occur with these techniques. First, extensive skin undermining is required for redraping the skin along the neck. This usually results in ligation of the subdermal attachments extending to the platysma. ${ }^{7}$ During rhytidectomy, the principle of placing tension on planes lying deep to the dermal layer has been a widely accepted theory. We believe that this theory also applies to the aging neck.

Corset techniques release the connections between the platysma and dermis, placing direct tension on the skin and addressing the muscle separately. With placement of a suture support spanning the submentum, the dermal-platysma ligamentous attachments are hooked by this subcutaneous suture, and the platysma and skin are pulled taught as a single unit within the neck.

Combining the percutaneous suspension suture and the posterior neck-lift techniques, we were able to also address prominent digastric muscles and ptotic submandibular glands, to some degree. The horizontal incision at the most inferior aspect of our platysmal flap provides further release, allowing us to provide additional contouring of the cervicomental angle, and addresses platysmal banding. The percutaneous suture also reinforces the lax platysma and keeps its edges from flaring out. As we developed our use of the percutaneous suture technique, we found it necessary to 
Table 1 Cephalometric Data Obtained from Subjects and Calculated Averages for Both Preoperative and Postoperative Values

\begin{tabular}{|c|c|c|c|c|}
\hline Subject & $\begin{array}{l}\text { Preoperative } \\
\text { CMA (Degrees) }\end{array}$ & $\begin{array}{l}\text { Postoperative } \\
\text { CMA (Degrees) }\end{array}$ & $\begin{array}{l}\text { Preoperative } \\
\text { HMD }(\mathrm{cm})\end{array}$ & $\begin{array}{l}\text { Postoperative } \\
\text { HMD }(\mathrm{cm})\end{array}$ \\
\hline 1 & 150.7 & 141.9 & 7.7 & 11.4 \\
\hline 2 & 129 & 115 & 12.2 & 11.8 \\
\hline 3 & 130.3 & 121.2 & 5.7 & 8 \\
\hline 4 & 131.5 & 1111.9 & 12.4 & 13.3 \\
\hline 5 & 139.1 & 113.9 & 3.7 & 11.1 \\
\hline 6 & 145.5 & 130.2 & 6.7 & 9.9 \\
\hline 7 & 126.1 & 118.6 & 9.9 & 9.1 \\
\hline 8 & 159.7 & 137.5 & 7.6 & 12.7 \\
\hline 9 & 114.3 & 106.6 & 11.9 & 12.2 \\
\hline 10 & 129.9 & 117.7 & 11.2 & 11.4 \\
\hline 11 & 138.2 & 96.1 & 12.9 & 15.3 \\
\hline 12 & 144.8 & 134.6 & 6.3 & 7.4 \\
\hline 13 & 103.4 & 101.9 & 9.9 & 11.1 \\
\hline 14 & 134.6 & 137.2 & 7.2 & 6.2 \\
\hline 15 & 136.4 & 128.2 & 8.8 & 10.9 \\
\hline 16 & 134.7 & 124.2 & 10.4 & 13 \\
\hline 17 & 121.3 & 110.1 & 10.6 & 8.5 \\
\hline 18 & 123.9 & 116.4 & 9.8 & 11 \\
\hline 19 & 131.7 & 119.8 & 12.9 & 9.6 \\
\hline 20 & 138.3 & 146.7 & 6.8 & 8.7 \\
\hline 21 & 136.5 & 124.2 & 7.4 & 10.3 \\
\hline 22 & 155.9 & 140.3 & 9.7 & 10.8 \\
\hline 23 & 127.5 & 131.4 & 4 & 4.7 \\
\hline 24 & 144 & 134.5 & 5.1 & 12.9 \\
\hline 25 & 142.5 & 110.5 & 2.8 & 8.7 \\
\hline \multirow[t]{3}{*}{ Averages } & 134.8 & 122.8 & 8.5 & 10.4 \\
\hline & & $\mathrm{CMA}$ reduction $=12.0$ & & $\mathrm{HMD}$ gain $=1.9 \mathrm{~cm}$ \\
\hline & $p=0.002$ & & $p=0.009$ & \\
\hline
\end{tabular}

CMA, cervicomental angle; HMD, hyomental distance.

incise across the platysma if any banding extended below the hyoid.

A key component of the procedure is the imbrication of the posterior/inferior edge of the platysma flap to the sternocleidomastoid fascia after pulling the posterior/superior edge of the platysma flap to the periosteum of the mastoid. We feel this prevents possible "window shading" of the platysma postoperatively. Within our cohort, one patient underwent a percutaneous suture technique without cutting the platysma. She wished to undergo revision surgery and we did so, employing the inferior separation and posterior pull of the platysma, with a satisfactory result.

The idea of spanning sutures within the neck for rejuvenation procedures was described by Webster et al. ${ }^{8}$ Their technique relied on sutures running below the border of the mandible to prevent the early recurrence of platysmal banding. Ptotic submandibular glands have been addressed with spanning sutures placed in the neck. ${ }^{9}$ The web neck "hammock" technique, employed by our practice, differs in that the sutures are placed percutaneously above the lower border of the mandible, thereby accentuating the mandible and the submentum while simultaneously addressing platysmal banding and ptotic submandibular glands.

A limitation of this study lies in its limited follow-up time (average 8 months for subjective and 1 month for objective outcomes). The aim of this study to was report our initial experiences. Surely, longer follow-up will be necessary to address whether our results hold over time and for how long. This will also allow us to possibly evaluate whether this technique is ideal for all patients or should be reserved for a subset of individuals. 

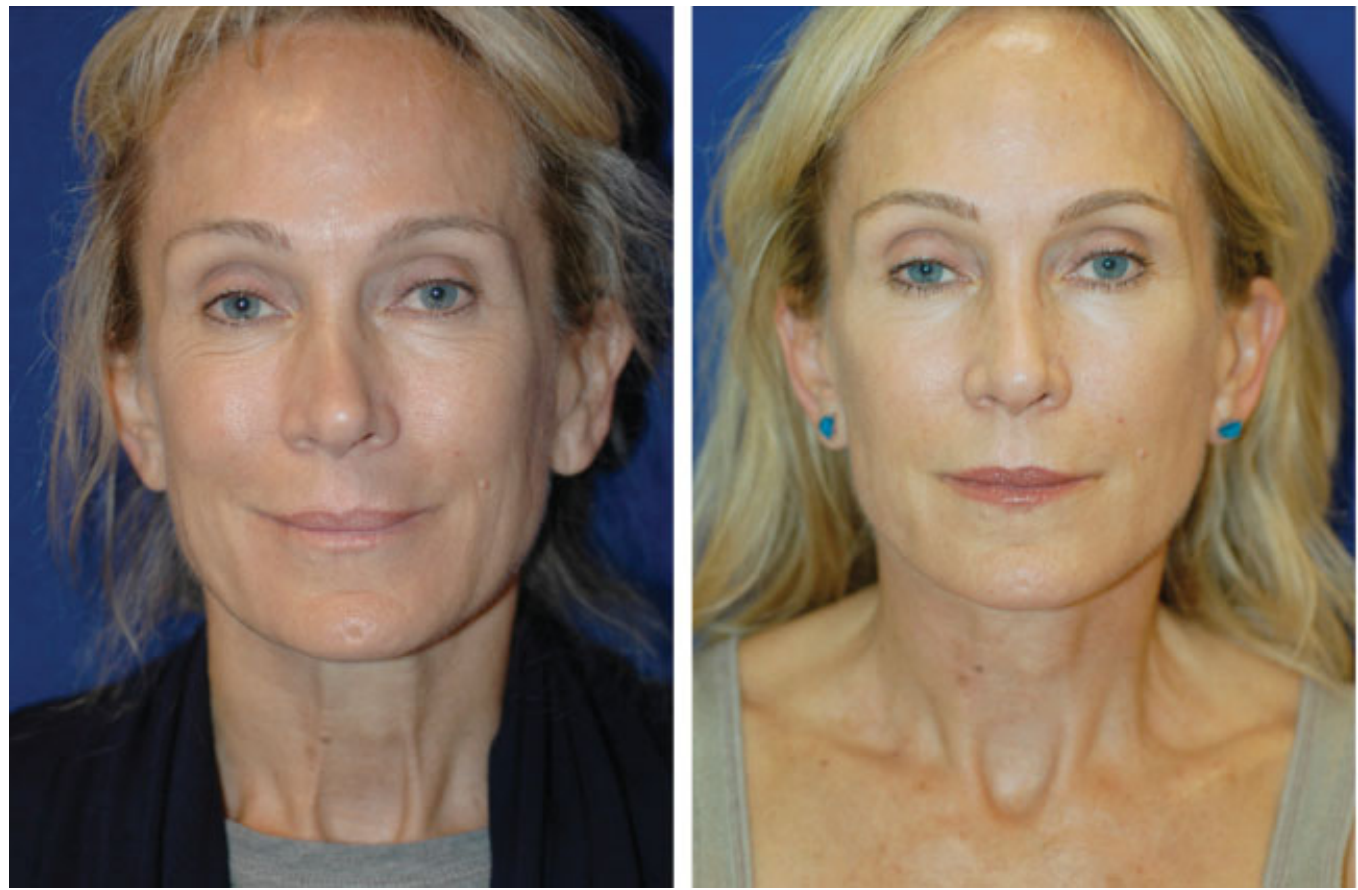

Figure 4 Preoperative (left) and postoperative (right) frontal views of a subject after treatment of her aging neck with our technique. She also underwent Ulthera therapy postoperatively to the face. @ Copyright 2011 Dr. Gregory S. Keller, M.D.

In this initial study, however, most results seemed to improve between 6 weeks and 6 months (-Fig. 8). The only longer-term deterioration was seen in the initial patient procedures, when a posterior platysmaplasty was not performed for indications described above.

Overall the procedure is well tolerated with patients, resulting in little to no downtime and readily performed under conscious sedation when used as an isolated procedure. Our operative time was significantly reduced-especial- ly important when used with concomitant rhytidectomy or other aging face procedures. The reduced morbidity of this technique allows easy incorporation into our operative plan.

\section{Conclusion}

We report our early experiences with use of a percutaneously placed spanning suture in the neck combined with a posterior platysmal pull. Outcomes have been favorable, due to its ease
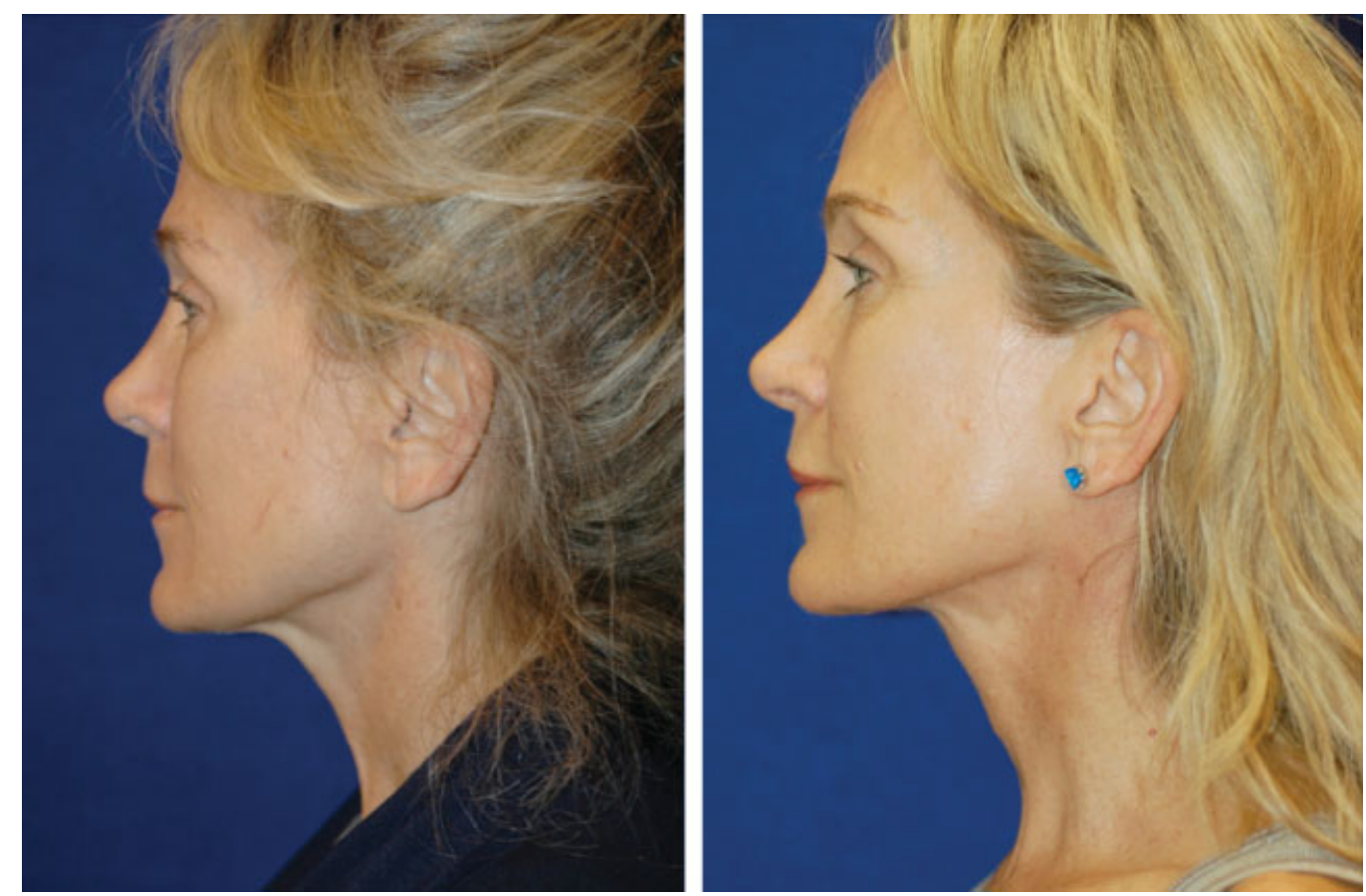

Figure 5 Preoperative (left) and postoperative (right) lateral views of the same subject. @ Copyright 2011 Dr. Gregory S. Keller, M.D. 

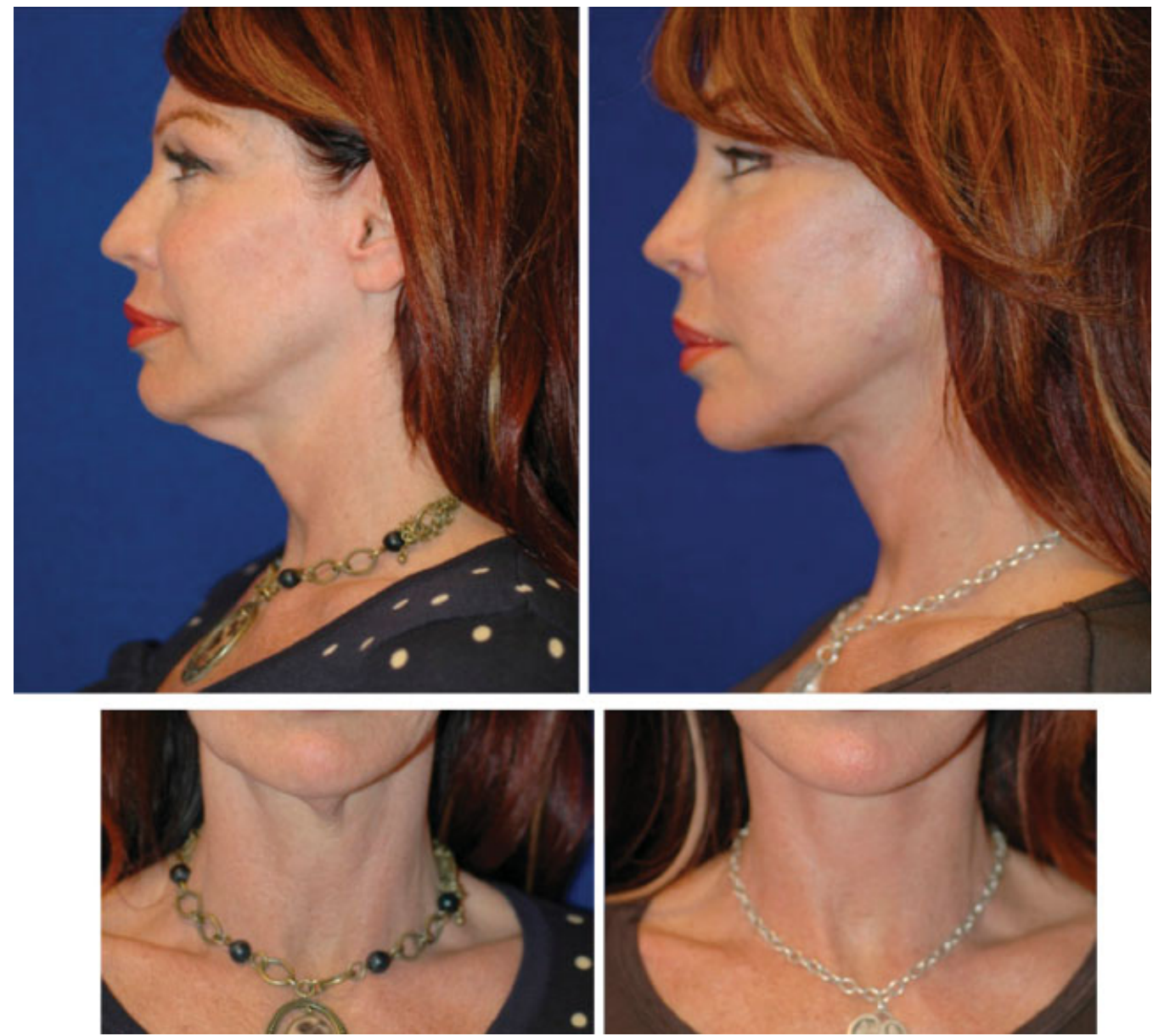

Figure 6 Preoperative (left) and postoperative (right) frontal and lateral views of a subject after treatment of her aging neck with our technique. She also underwent revision rhytidectomy at the time of procedure. @ Copyright 2011, Dr. Gregory S. Keller, M.D.
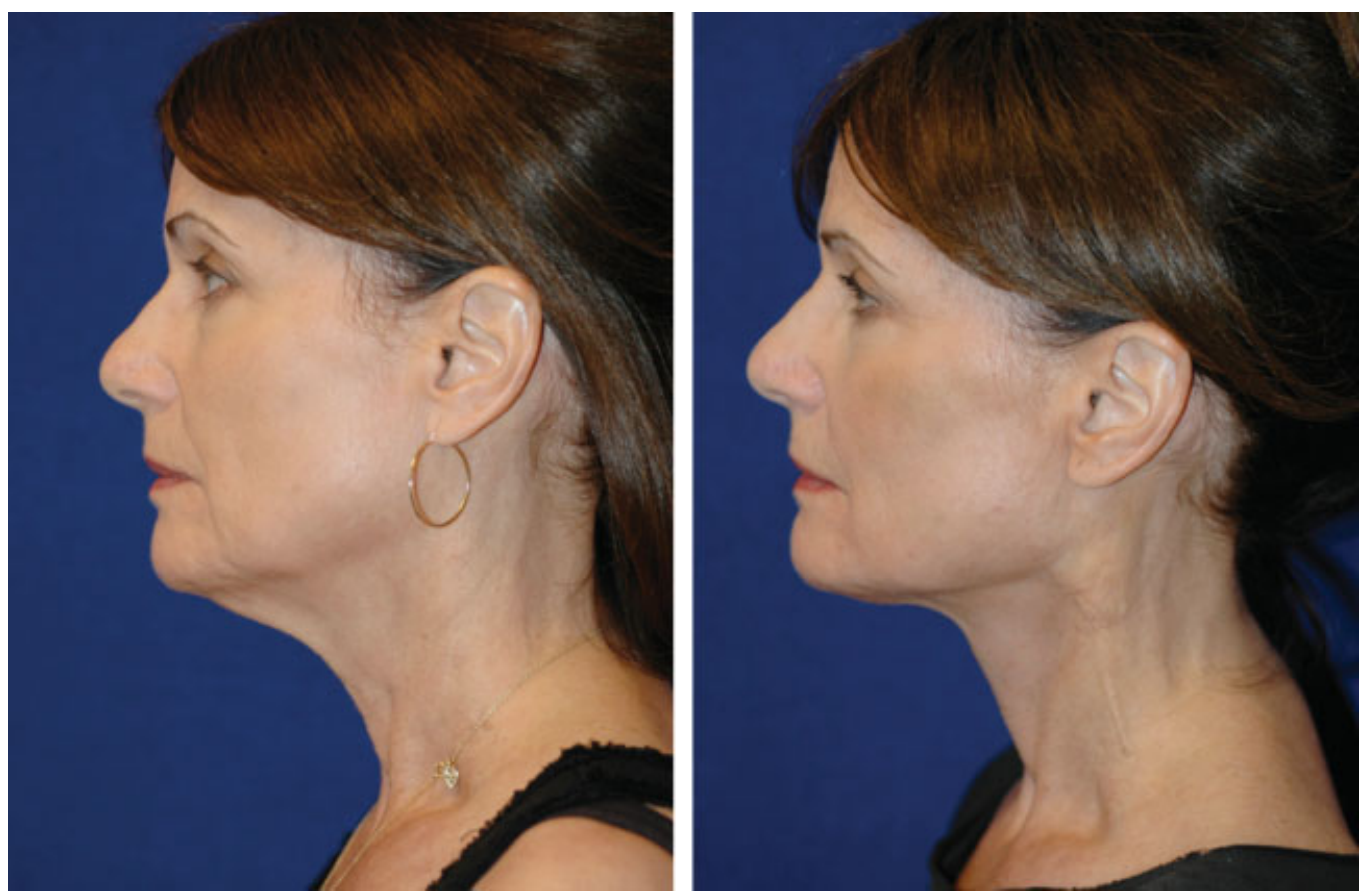

Figure 7 Preoperative (left) and 4-month postoperative (right) lateral views of a subject after our technique with concomitant rhytidectomy. (c) Copyright 2011 Dr. Gregory S. Keller, M.D. 

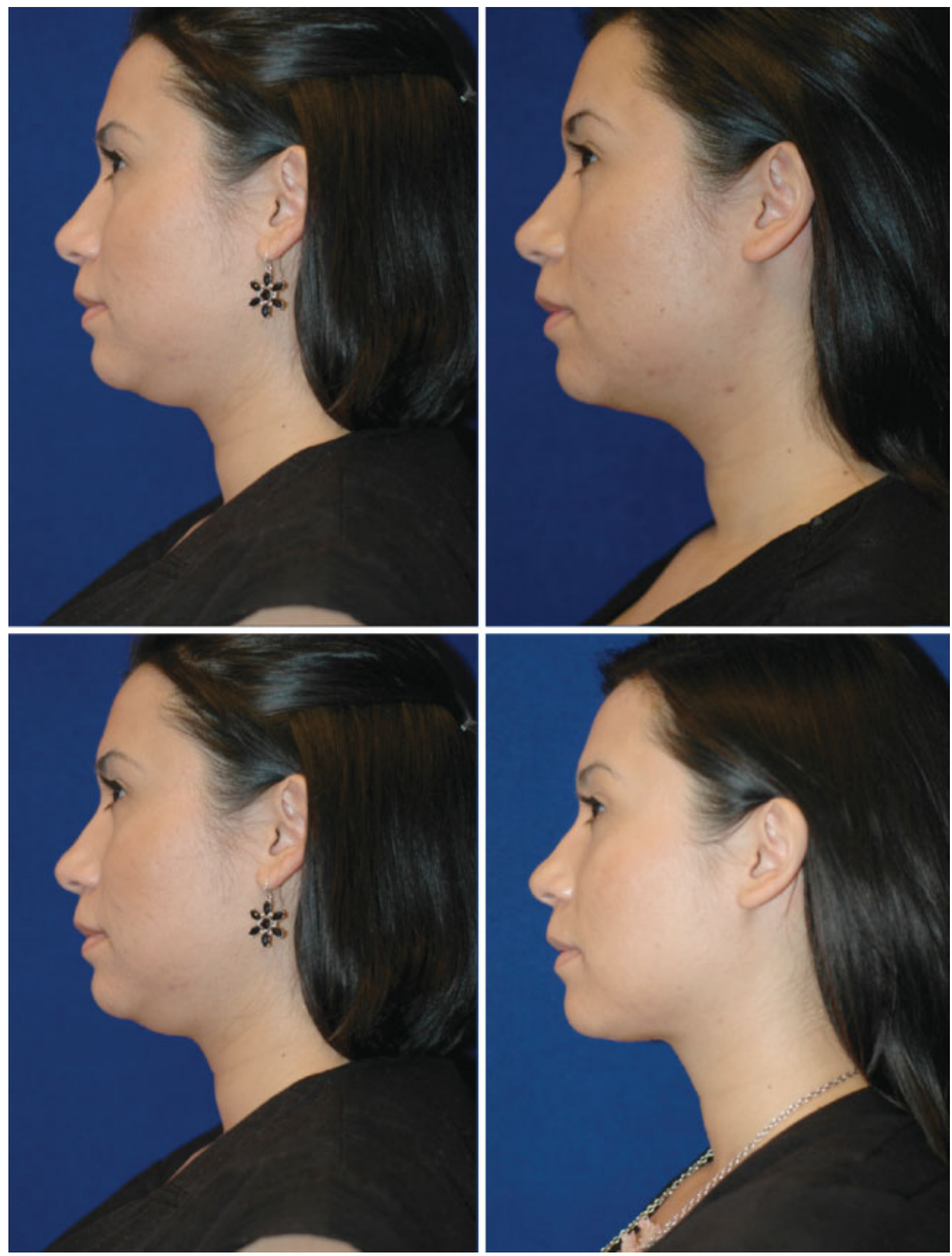

Figure 8 Preoperative (left) and 6-week (upper right) and 6-month (lower right) postoperative lateral views. A few subjects demonstrated additional improvement over time. (c) Copyright 2011 Dr. Gregory S. Keller, M.D.

of use and reproducibility, minimal downtime, and ability to address the many facets of the aging neck simultaneously.

Although our initial experiences are very favorable, further long-term studies are warranted to validate our initial success with this procedure. Until then, it represents a useful "tool" to add to the aesthetic surgeon's armamentarium to treat the sagging neck.

\section{Note}

Presented at The American Academy of Facial Plastic and Reconstructive Surgery Rejuvenation of the Aging Face Meeting, San Diego, California, January 22, 2011.

\section{References}

1 Feldman JJ. Corset platysmaplasty. Plast Reconstr Surg 1990; 85:333-343

2 Mashkevich G, Wang J, Rawnsley J, Keller GS. The utility of ultrasound in the evaluation of submental fullness in aging necks. Arch Facial Plast Surg 2009;11:240-245

3 Doerr TD. Lipoplasty of the face and neck. Curr Opin Otolaryngol Head Neck Surg 2007; 15:228-232

4 Bitner JB, Friedman O, Farrior RT, Cook TA. Direct submentoplasty for neck rejuvenation. Arch Facial Plast Surg 2007;9: 194-200

5 www.blackandblacksurgical.com/iguide.php. Accessed February 16,2012 
134 The Web Lift and Posterior Pull for the Aging Face Ezzat et al.

6 Connell BF. Neck contour deformities. The art, engineering, anatomic diagnosis, architectural planning, and aesthetics of surgical correction. Clin Plast Surg 1987;14:683-692

7 Fogli AL. Skin and platysma muscle anchoring. Aesthetic Plast Surg 2008;32:531-541
8 Webster RC, Smith RC, Smith KF. Face lift, part 5: suspending sutures for platysma cording. Head Neck Surg 1984;6:870-879

9 Giampapa VC, Di Bernardo BE. Neck recontouring with suture suspension and liposuction: an alternative for the early rhytidectomy candidate. Aesthetic Plast Surg 1995;19:217-223 